\title{
Caranguejos e Camarões (Crustacea: Decapoda) do Igarapé do Perdido, Apiaú, Mucajaí, Roraima
}

\author{
Patrícia Macedo de Castro ${ }^{1,2}$ \& Marliane Rodrigues da Silva ${ }^{1,3}$
}

1. Museu Intergado de Roraima, Instituto de Amparo a Ciência, Tecnologia e Inovação do Estado de Roraima/IACTIRR. Av. Brigadeiro Eduardo Gomes, 2868, Parque Anauá, Bairro dos Estados, Boa Vista, RR, CEP 69.305-010.

2. Universidade Estadual de Roraima - UERR, Programa de Pós-Graduação em Ensino de Ciências. Av. Sete de Setembro, 231, Canarinho, Boa Vista, RR, CEP 69.306-530. Autor para contato: pmcfemact@gmail.com.br 3. Faculdade Cathedral. Av. Luis Canuto Chaves, 293, Bairro Caçari, Boa Vista, RR, CEP 69307-053.

Recebido em : 25/02/2013. Aceito em: 01/03/2013.

\section{RESUMO}

O presente trabalho teve por objetivo verificar a diversidade e abundância dos crustáceos decápodas presentes no igarapé do Perdido, localizado na região do Apiaú, município de Mucajaí, estado de Roraima. As coletas foram realizadas em agosto e setembro de 2009, e nos meses de março e agosto de 2010, sendo coletados 248 caranguejos e camarões durante o período amostral. A família Palaemonidae foi representada por seis espécies do gênero Macrobrachium e a família Trichodactylidae por duas espécies pertencentes aos gêneros Sylviocarcinus e Valdivia. O gênero Macrobrachium foi dominante contribuindo com $94 \%$ do total dos indivíduos coletados. As espécies $M$. brasiliense, $M$. inpa e $V$. serrata são registradas pela primeira vez para o estado de Roraima.

PALAVRAS CHAVE: Brachyura, Palaemonidae, Trichodactylidae.

\section{ABSTRACT}

The present work had the goal of verifying the diversity and abundance of decapod crustaceans present in the Igarapé Perdido, located in the region of Apiaú, county of Mucajaí, Roraima State. The gatherings were conducted on August and September of 2009, and on the months of March and August of 2010, being collected 248 crabs and shrimps during the sample period. The Palaemonidae family was represented by six species of the Macrobrachium genus and the Trichodactylidae family by two species belonging to the Sylviocarcinus and Valdivia genus. The Macrobrachium genus was dominant contributing with $94 \%$ of the total of collected individuals. The species M. brasiliense, M. inpa and V. serrata are registered for the first time to the Roraima state.

KEYWORDS: Brachyura, Palaemonidae, Trichodactylidae.

\section{INTRODUÇÃO}

A Amazônia possui cerca de dois a quatro quilômetros lineares de igarapés por $\mathrm{Km}^{2}$ de floresta e abriga uma biota pouco conhecida e diversa, que vem sofrendo impactos ambientais de várias origens (Walker 1995 apud Goch 2006).

Os igarapés abrigam conjuntos únicos de espécies, que são progressivamente substituídas ao longo da rede de drenagem e contribuem significativamente para a diversidade regional. Além das espécies que tipicamente ocupam estes ambientes, espécies não residentes utilizam frequentemente estes corpos d'água como áreas de alimentação, sítios para reprodução, recuperação ou refúgio, sendo, portanto, ambientes importantes para uma vasta diversidade de organismos (Anjos 2007).

Os crustáceos decápodas têm grande importância nos processos ecológicos dos ambientes aquáticos, pois atuam em diferentes níveis da cadeia trófica desses ambientes, quer como herbívoros, predadores, necrófagos ou presas de outros grupos, além disso, os crustáceos são importantes na caracterização da saúde ambiental, pois funcionam como bioindicadores de qualidade ambiental (Vieira 2003).

A ordem Decapoda está representada na região Amazônica pelas infraordens Caridea e Brachyura, as quais são compostas pelos camarões e caranguejos dulcícolas, sendo elementos importantes da biota aquática de riachos, rios, igarapés, lagos e açudes, tanto pelo aspecto ecológico quanto pelo econômico (Brusca \& Brusca 2003; Magalhães 2003; Neto 2007).

A infraordem Caridea compreende os camarões que constituem um grupo bem diversificado, tanto com relação ao seu hábitat quanto a sua morfologia. No Brasil, somente as famílias Sergestidae, Atyidae e Palaemonidae apresentam espécies que ocorrem em ambientes de água doce (Melo 2003).

As famílias Sergestidae e Palaemonidae apresentam uma grande diversidade de espécies nos corpos de água da bacia Amazônica. Visto 
aos inúmeros microhábitats existentes em função da extensa drenagem do rio Amazonas, sendo que a família Palaemonidae é a que apresenta maior diversidade, abundância e interesse econômico pela potencialidade que apresenta para o cultivo (Melo 2003; Vieira 2003).

$\mathrm{Na}$ infraordem Brachyura, encontra-se os caranguejos dulcícolas pertencentes à superfamília Potamoidea (sensu Bowman \& Abele, 1982) que, no Novo Mundo, está representada pelas famílias Pseudothelphusidae e Trichodactylidae, ambas ocorrendo no Brasil, com $60 \%$ das espécies atualmente conhecidas distribuídas na bacia Amazônica (Magalhães 2003). São exclusivamente dulcícolas, ou seja, cumprem todo o seu ciclo de vida em águas continentais, sem qualquer dependência de águas marinhas ou estuarinas (Magalhães 1998; 1999; 2003).

Os camarões de água doce devido a sua importância para o consumo humano, bem como seu papel de espécies chaves em comunidades aquáticas da Amazônia merece destaque como indicadores de funcionamento de ecossistemas. As espécies são componentes importantes na rede alimentar aquática, sendo também encontradas no conteúdo estomacal de peixes em muitas partes da Amazônia (Overal 2001).

Informações sobre a diversidade, endemismo, distribuição e outros aspectos biológicos em ambientes aquáticos são extremamente importante através de projetos de inventariamento da fauna de crustáceos decápodas, assim como, estudos de taxonomia, sistemática e ecologia. $\mathrm{O}$ conhecimento da diversidade biológica na bacia Amazônica, especialmente na bacia do rio Branco ainda é muito incipiente, principalmente nas regiões de cabeceiras dos igarapés, onde o grau de endemismo pode ser elevado. Sendo assim, o presente trabalho teve por objetivo verificar a diversidade e abundância dos crustáceos decápodas presentes no igarapé do Perdido, localizado na região do Apiaú, município de Mucajaí, estado de Roraima.

\section{MATERIAL E MÉTODOS}

O estudo foi realizado no igarapé do Perdido $\left(02^{\circ} 35^{\prime} 39,9^{\prime \prime} \mathrm{N}\right.$ e $61^{\circ}, 05^{\prime}, 02,3$ ' $\left.\mathrm{W}\right)$, na região do Apiaú, no município de Mucajaí, estando a $70 \mathrm{~m}$ de altitude em relação ao nível do mar, o qual possui um clima quente $\mathrm{e}$ úmido, e temperatura variando entre $20^{\circ}$ a $35^{\circ} \mathrm{C}$ (Freitas 1998). A cidade é originária da antiga Colônia Agrícola do Mucajaí, fundada em 1951, nomeada de Colônia Agrícola Fernando Costa e suas principais vilas são Apiaú e Tamandaré (Freitas 1998).

As coletas foram realizadas em agosto e setembro de 2009, e nos meses de março e agosto de 2010. Os camarões e caranguejos foram coletados com puçá passado junto à vegetação aquática, na serrapilheira submersa, margens das barrancas e ocos de paus submersos, e fixados em álcool a 70\%.

Em laboratório, após a triagem, os caranguejos e camarões foram determinados e sexados (machos, fêmeas, fêmeas ovígeras e jovens) com o auxílio de estereomicroscópio. A largura da carapaça dos caranguejos e o comprimento do cefalotórax dos camarões foram mensurados com paquímetro digital com precisão de $0,02 \mathrm{~mm}$. Os espécimes coletados foram depositados na Coleção Zoológica do Museu Integrado de Roraima (CRUST MIRR) distribuídos por espécie nos seguintes lotes: MIRR 053; MIRR 056; MIRR 058; MIRR 059; MIRR 060; MIRR 079; MIRR 080; MIRR 081; 206 MIRR; 207 MIRR; 208 MIRR; 209 MIRR; 210 MIRR; 212 MIRR; 213 MIRR; 215 MIRR; 216 MIRR; 217 MIRR; 218 MIRR; 219 MIRR; 220 MIRR; 221 MIRR; 222 MIRR; 223 MIRR; 234 MIRR; 235 MIRR; 236 MIRR; 237 MIRR; 238 MIRR; 239 MIRR; 240 MIRR; 241 MIRR; 242 MIRR; 243 MIRR.

Para a verificação da diversidade e abundância das espécies de crustáceos decápodas foi utilizado o índice de ShannomWiener (Krebs, 1999) com a fórmula:

$H^{\prime}=-\sum$ (PI) (ln pi)

Onde: pi - representa a proporção de exemplares da i-ésima espécie.

Como em uma amostra o verdadeiro valor de pi é desconhecida, o pi é estimado pela seguinte fórmula:

$\mathbf{p i}=(\mathbf{n i} / \mathbf{N})$

Onde:

ni é o número de exemplares pertencentes à espécie "i";

$\mathbf{N}$ é o número total de exemplares coletados na amostra.

Para calcular a abundância relativa (equitabilidade) das espécies de crustáceos decápodas, foi utilizado o cálculo de abundância relativa (Magurran 1988) o qual propõe a relação da diversidade observada e a diversidade máxima, sendo expressa pela 
fórmula:

\section{$\mathbf{E}=\mathbf{H}^{\prime} / \ln \mathbf{S}$}

Onde: H' é o índice de diversidade de Shannon-Wiener;

$\mathbf{S}$ é o número de espécies presentes na amostra.

\section{RESULTADOS E DISCUSSÃO}

Foram coletados 248 crustáceos decápodas no igarapé do Perdido durante todo o período amostral (Tabela 1). O número de camarões da família Palaemonidae totalizou 235 indivíduos pertencentes ao gênero Macrobrachium. Os caranguejos coletados pertencem à família Trichodactylidae, totalizando 11 indivíduos do gênero Sylviocarcinus e dois espécimes do gênero Valdivia.

A família Palaemonidae foi representada por seis espécies, sendo Macrobrachium brasiliense (Heller, 1862), Macrobrachium inpa Kenley e Walker, 1982, Macrobrachium nattereri (Heller, 1862), Macrobrachium spl, Macrobrachium sp2 e Macrobrachium sp3 (Tabela 1).

Segundo Sampaio (2009), o gênero Macrobrachuim, é dominante e o mais representativo, onde muitas espécies desse gênero são utilizadas para carcinicultura e tem sido frequentemente encontradas em habitats aquáticos naturais do país. No igarapé do
Perdido, o gênero Macrobrachuim foi dominante contribuindo com $94 \%$ do total dos crustáceos decápodas coletados. A espécie de camarão que apresentou maior representatividade foi Macrobrachuim spl e das espécies de caranguejo foi Sylviocarcinus pictus. Com relação a ocorrência, somente as espécies Macrobrachium brasiliense e Macrobrachium spl foram capturadas durante todo o período amostral nas quatro coletas realizadas nos anos de 2009 e 2010, abrangendo estação seca e chuvosa.

As espécies de camarões denominadas Macrobrachium sp1, Macrobrachium sp2 e Macrobrachium sp3 foram classificadas somente a nível de gênero pela dificuldade em determinar o táxon a nível de espécie, principalmente devido ao pequeno tamanho dos espécimes capturados, dificultando a visualização de caracteres típicos dos machos adultos. Como os adultos tendem a permanecer escondidos durante o dia embaixo de pedras, troncos submersos e dentro de tocas, enquanto as formas juvenis e imaturos são mais facilmente encontradas junto à vegetação aquática e a serrapilheira submersa. Além disso, a taxonomia dos camarões de água doce apresenta algumas dificuldades relacionadas principalmente à ocorrência de dimorfismo sexual e a semelhança morfológica entre alguns complexos de espécies (Sampaio et al. 2009).

Tabela 1 - Espécies de crustáceos decápodas capturados durante o período amostral de agosto e setembro de 2009 e março e agosto de 2010, classificados por família, gênero e espécie coletados no igarapé do Perdido, Apiaú, Mucajaí, RR.

\begin{tabular}{|c|c|c|c|c|c|}
\hline \multirow{2}{*}{$\begin{array}{l}\text { CLASSIFICAÇÃO } \\
\text { Família } \\
\text { Espécie }\end{array}$} & \multicolumn{4}{|c|}{ COLETAS } & \multirow{2}{*}{$\begin{array}{l}\mathrm{N}^{\circ} \text { TOTAL DE } \\
\text { INDIVÍDUOS }\end{array}$} \\
\hline & 1 & 2 & 3 & 4 & \\
\hline \multicolumn{6}{|l|}{ PALAEMONIDAE Rafinesque, 1815} \\
\hline \multicolumn{6}{|l|}{ Macrobrachium Bate, 1868} \\
\hline Macrobrachium brasiliense (Heller, 1862) & $\mathrm{X}$ & $\mathrm{X}$ & $\mathrm{X}$ & $\mathrm{X}$ & 62 \\
\hline Macrobrachium inpa Kensley \&Walker, 1982 & & $\mathrm{X}$ & & $\mathrm{X}$ & 9 \\
\hline Macrobrachium nattereri (Heller, 1862) & $\mathrm{X}$ & $\mathrm{X}$ & & & 32 \\
\hline Macrobrachium spl & $\mathrm{X}$ & $\mathrm{X}$ & $\mathrm{X}$ & $\mathrm{X}$ & 129 \\
\hline Macrobrachium sp2 & $\mathrm{X}$ & $\mathrm{X}$ & & & 1 \\
\hline Macrobrachium sp3 & $\mathrm{X}$ & & & & 2 \\
\hline \multicolumn{6}{|l|}{ TRICHODACTYLIDAE H. Milne-Edwards, 1853} \\
\hline \multicolumn{6}{|l|}{ Sylviocarcinus H. Milne-Edwards, 1853} \\
\hline Sylviocarcinus pictus (H. Milne-Edwards, 1853) & & $X$ & & $\mathrm{X}$ & 11 \\
\hline \multicolumn{6}{|l|}{ Valdivia White, 1847} \\
\hline Valdivia serrata White, 1847 & $\mathrm{X}$ & & & & 2 \\
\hline TOTAL & & & & & 248 \\
\hline
\end{tabular}


A família Trichodactylidae apresentou duas espécies coletadas de gêneros distintos, sendo Sylviocarcinus pictus (H. MilneEdwards, 1853) e Valdivia serrata White, 1847 (Tabela 1). Os representantes da família Trichodactylidae estão presentes em praticamente todas as bacias hidrográficas do território nacional, grupo típico de rios de planícies, sendo raras as espécies que habitam corpos d'água situados em altitudes superiores a 300 metros (Magalhães 2003).

Representantes da família Pseudothelphusidae não foram coletados, pois são caranguejos de regiões montanhosas, geralmente vivendo em áreas entre 300 e 3.000 metros de altitude, a grande maioria das espécies tem área de distribuição bastante reduzida, sendo normalmente encontradas em rios provenientes dos Maciços das Guianas e do Brasil Central, ou então em pequenos igarapés na floresta de terra firme, não sendo encontradas em regiões alagáveis (Rodríguez 1981; Magalhães 2003).

A abundância relativa (E) dos crustáceos decápodas foi verificada para o período amostral (Tabela 2). Comparando as quatro coletas realizados no igarapé do Perdido, foi observado uma grande abundância de

Tabela 2 - Abundância relativa das espécies de crustáceos decápodas coletados no igarapé do Perdido, Apiaú,

Mucajaí, RR.

\begin{tabular}{ll}
\hline ESPÉCIES & ÍNDICE DE SHANNON \\
& ABUNDÂNCIA (E) \\
\hline M. brasiliense & $\mathrm{E}=1,13$ \\
M. inpa & $\mathrm{E}=0,02$ \\
M. nattereri & $\mathrm{E}=0,30$ \\
M. sp1 & $\mathrm{E}=4,16$ \\
M. sp2 & $\mathrm{E}=0$ \\
M.sp3 & $\mathrm{E}=0$ \\
S. pictus & $\mathrm{E}=0,04$ \\
V. serrata & $\mathrm{E}=0$ \\
\hline
\end{tabular}

indivíduos capturados do gênero

Macrobrachium.

Em relação à abundância, a concentração do número de crustáceos decápodas em apenas três gêneros pode estar relacionada às características específicas do habitat e também relacionada a inúmeros fatores, como áreas antropizadas (Magalhães \& Pereira 2007). Parte do igarapé do Perdido fica situado em fazenda onde há criação de gado e piscicultura, apresentando em alguns pontos a mata ciliar alterada. Porém, os espécimes sempre foram coletados junto a vegetação marginal das barrancas, serrapilheira submersa e ocos de paus submersos.

Dos crustáceos decápodas coletados durante o período de coleta, foi observado uma predominância de fêmeas (149), seguidas pelos machos (53) e jovens (46). As espécies $M$. spl e $M$. brasiliense foram as que apresentaram o maior número de fêmeas capturadas durante todo o período amostral, podendo estar relacionado com a sazonalidade local, comportamento e o período reprodutivo, o qual ocorre após o período de intensas chuvas ou inverno regional. No estado de Roraima as temperaturas são elevadas, com pouca flutuação durante todo o ano, sendo o que diferencia as estações é a distribuição das chuvas ao longo do ano, com inverno chuvoso e verão seco. A maioria das coletas foram realizadas no período chuvoso que se caracteriza de abril a agosto e somente a coleta no mês de março foi realizada no período seco.

A Figura 1 apresenta a distribuição da frequência absoluta dos crustáceos decápodas coletados ao longo do período amostral classificados em machos, fêmeas e jovens. As fêmeas foram coletados em quase todos as espécies com exceção de $M . s p 2, M . s p 3$ e $V$. serrata, onde foram capturados apenas jovens fato que dificultou a determinação em nível de espécie, principalmente por ocorrência de dimorfismo sexual e semelhança morfológica de algumas espécies principalmente quando

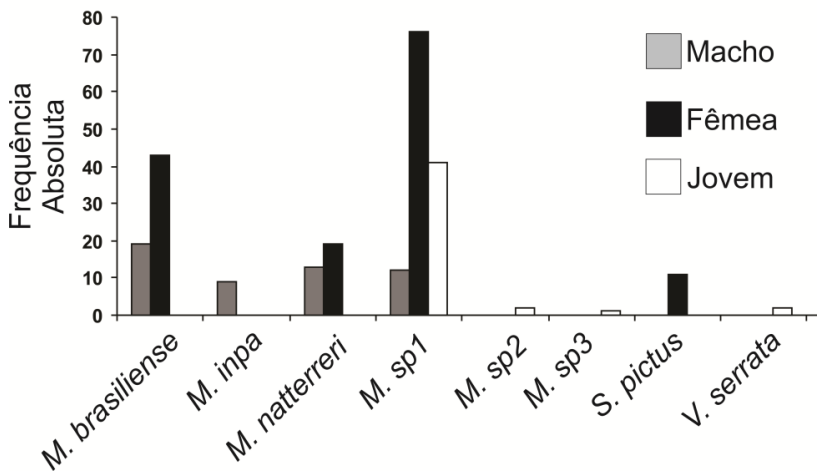

Figura 1 - Distribuição frequência absoluta das espécies de crustáceos decápodas classificados em macho, fêmea e jovem coletados no igarapé do Perdido, Apiaú, Mucajaí, RR.

jovens (Sampaio 2009).

Foi verificada uma alta diversidade de crustáceos decápodas no igarapé do Perdido, devido ao elevado número de espécies de camarões e caranguejos capturados, sendo 
registradas seis espécies distribuídas no gênero Macrobrachium e duas espécies de caranguejos nos gêneros Sylviocarcinus e Valdivia. São registradas pela primeira vez para o estado de Roraima as espécies $M$. brasiliense, $M$. inpa e V. serrata.

\section{AGRADECIMENTOS}

Ao $\mathrm{CNPq}$ pelo apoio financeiro referente ao Processo 35.0413/2005-3-DCR/CNPq/ FEMARH. Ao Laboratorista do MIRR Anderson Alberto Otaviano e aos acadêmicos do curso de Ciências Biológicas da UERR pelo apoio nas coletas: Francinéia Zanetti da Costa, Dizoneide de Almeida Lima, Jusciléia Tavares da Silva e Adomeu de Sousa Sales.

\section{REFERÊNCIAS BIBLIOGRÁFICAS}

Anjos, H.D.B. Efeitos da fragmentação florestal sobre as assembléias de peixes de igarapés de zona urbana de Manaus, Amazonas. Dissertação de Mestrado, Universidade Federal do Amazonas, 2007.

Brusca, R.C.; Brusca, G. J. Invertebrados. 2007. Guanabara Koogan, Rio de Janeiro.

Freitas, A. Estudos Sociais - Roraima: Geografia e História. 1998. $5^{\mathrm{a}}$ ed. Corprint Gráfica e Editora Ltda, São Paulo.

Gárcia-Dávila, C.R.; Magalhães, C. 2003. Revisão taxonômica dos camarões de água doce (Crustacea: Decapoda: Palamonidae, Sergestidae) da Amazônia Peruana. Acta Amazônica 33(4): 663-686.

Goch, Y.G.F. Efeitos de assoreamento versus abundância de peixes e camarões em tributários do rio urucu localizados na base de operações da Petrobras "geólogo Pedro de Moura", Coari - AM. Tese de Doutorado, Instituo Nacional de Pesquisa da Amazônia, 2006.

Krebs, C.J. 1999. Ecological Methodology. Harper Collins Publichers, New York.

Magalhães, C. 1986. Revisão taxonômica dos caranguejos de água doce brasileiros da família Pseudothelphusidae (Crustacea, Decapoda). Amazoniana IX - 4: 609-623.

Magalhães, C. 1998. Malacostraca - Eucarida. Brachyura. Pseudothelphusidae and Trichodactylidae. In: Young, P.S. (Ed.). Catalague of Crustacea of Brazil. Museu Nacional, Rio de Janeiro, p. 517-523. [Série Livros n. 6].

Magalhães, C. 1999. Família Trichodactylidae (caranguejos braquiúros de água doce). In: Buckup, L \& Bond-Buckup, G. (Eds.). Os crustáceos do Rio Grande do Sul. Ed. Universidade/UFRGS, Porto Alegre, p. 486-490.
Magalhães, C. 2003. Famílias Pseudothelphusidae e Trichodactylidae. In: Melo, G.A.S. (Ed.). Manual de identificação dos Crustacea Decapoda de água doce do Brasil. Edições Loyola, São Paulo, p. 143-288.

Magalhães, C.; Pereira, G. 2007. Avaliação da diversidade de crustáceos decápodos na região do Escudo das Guianas com o objetivo de decisões de conservação. Biota Neotropical. 7(2): 111-129.

Magurran, A.E. 1988. Ecological Diversity and Its Measure. Chapman and Hall, New York, 215 p.

Melo, G.A.S. Manual de identificação dos crustácea decápoda de água doce do Brasil. 2003. Editora Loyola, São Paulo, 430p.

Neto, E.M.C. 2007. O caranguejo de água doce Trichodactylus fluviatilis (Latreille, 1828), (Crustacea, Decapoda, Trichodactylidae), na concepção dos moradores do povoado de Pedra Branca, Bahia, Brasil. Biotemas, 20(1): 59-68.

Overal, W.L. 2001. O peso dos invertebrados na balança de conservação biológica da Amazônia. In: Veríssimo, A. (Ed). Biodiversidade na Amazônia Brasileira. Estação Liberdade e Instituto Socioambiental, São Paulo, p. 50-59.

Rodríguez, G. 1981. Decapoda. In: Hulbert, S.H.; Rodríguez, G. \& Santos, N.D. (Eds.). Aquatic Biota of Tropical South America. Part 1: Arthropoda. San Diego States University, San Diego, p. 41-51.

Sampaio, S.R.; Nagata, J. K.; Lopez, O. L.; Masunari, S. 2009. Camarões de águas continentais (Crustacea, Caridea) da Bacia do Atlântico oriental paranaense, com chave de identificação tabular. Acta Biológica do Paraná, 38(1-2): 11-34.

Vieira, I.M. Diversidade de Crustáceos das Ressacas das Lagoa dos Índios, Tacacá e APA do Curiaú. 2003. In: Takiyana, L.R.; Silva, A.Q. (Orgs.). Diagnóstico das Ressacas do Estado do Amapá: Bacias do Igarapé da Fortaleza e rio Curiaú, Macapá, CPAQ/IEPA e DGEO/SEMA. p.53-62. 